\title{
FDS and Abaqus Coupling Toolkit for Fire Simulation and Thermal and Mass Flow Prediction
}

\author{
LIGUO CHEN, CHANGSONG LUO, and JIM LUA \\ Global Engineering and Materials, Inc. \\ 1 Airport Place, Suite 1 \\ Princeton, NJ 08540 USA
}

\begin{abstract}
Driven by the need for high performance and reduction of the ownership cost of commercial and military structures, both composite and aluminum materials have been used extensively. Protection of these advanced structures from fire is a critical issue in structural design and certification. Current limited analysis capability of commercial tools has forced the design to rely on a large number of component and structural level fire tests of a pre-loaded structure. The main focus of this research is to develop an Abaqus Fire Interface Simulator Toolkit (AFIST) by integration of a customized Fire Dynamics Simulator (FDS) with a customized Abaqus structural analyzer through a two-way coupling. A real fire environment is simulated using FDS and its impact on the thermal-mechanical response of the structure is fully captured by Abaqus. Exchange of heat and mass at the coupling interface is achieved based on the averaged energy and mass conservation operating over a given spatial and temporal domain. Demonstration and validation examples are presented to illustrate the tool capability for simulating the multiple coupling interfaces, flame spread, and thermal and mass diffusion. The simulation results from AFIST are compared with the prediction from the standalone FDS and experimental data published in the literature.
\end{abstract}

KEYWORDS: CFD, structural response, modeling, heat transfer, fluid dynamics, flame spread.

\section{NOMENCLATURE LISTING}

A pre-factor of Arrhenius law $\left(\mathrm{s}^{-1}\right)$

$k \quad$ thermal conductivity $(\mathrm{W} / \mathrm{m} \cdot \mathrm{K})$

$p_{g} \quad$ gas pressure $(\mathrm{Pa})$

$c \quad$ thermal capacity $(\mathrm{kJ} / \mathrm{kg} \cdot \mathrm{K})$

$h$ enthalpy $\left(\mathrm{kJ} / \mathrm{m}^{3}\right)$

$E \quad$ activation energy $(\mathrm{J} / \mathrm{mol})$

$R \quad$ gas constant $(\mathrm{J} / \mathrm{K} \cdot \mathrm{mol})$

$L \quad$ glass thickness (m)

$l$ decay length $(\mathrm{m})$

$q$ heat flux $\left(\mathrm{kW} / \mathrm{m}^{2}\right)$

$s \quad$ shaded length $(\mathrm{m})$

$T_{o} \quad$ ambient temperature $(\mathrm{K})$

$T$ temperature (K)

$t \quad$ time (s)

Greek

$\gamma \quad$ emissivity

$\varepsilon \quad$ strain

$\rho \quad$ density $\left(\mathrm{kg} / \mathrm{m}^{3}\right)$

$\sigma \quad$ stress, Stefan-Boltzmann constant

\section{INTRODUCTION}

Unconventional materials such as composites and aluminum alloys have been increasingly used as military and commercial building materials. Polymer matrix composites (PMCs) are used over a wide range of applications in marine, aerospace, civil infrastructure, and automobiles because of their high specific strength, long fatigue life, and excellent corrosion resistance. Although PMCs have lower thermal conductivity than conventional metals, thus preventing the spread of fire from the heat source, they have worse overall fire performance because of the fire-induced chemical and mechanical degradation in PMCs [1-4]. The dense smoke, soot, and toxic gases generated from the decomposition reaction for PMCs in fire increase the health risk and decrease the survivability for anyone remaining in the area. The reduction in strength of heated composites, especially compression strength, will have a large impact on the structural integrity and its final rupture.

Fire simulations of structural response and failure predictions are a challenging multi-physics problem. The key phenomena associated with the thermal, chemical, and mechanical processes are shown in Fig. 1. The thermal process involves heat conduction, convection, heat generation, and radiation associated with the heat source, external flow, reaction gas, and volatile and moisture flow. The chemical reaction and material 
degradation of a FRP consists of pyrolysis and core volatilization of the polymer resin and growth and oxidation of char. These physical processes interact with each other resulting in: 1) delamination induced insulation; 2) pore pressure accelerated delamination; and 3) perturbation of near-surface flow from the deformed shape. In addition, it is still questionable if the fire phenomena and structural damage are scalable. Given the problem complexity and uncertainty in applying the laboratory test data for the design of fire resistant structures, full-scale tests are still used heavily for the design certification of the final structures despite the costly and time consuming process. It is imperative to develop a physics based fire simulation and damage assessment tool to replace the current prescriptive approach with a performancebased analysis procedure that will give designers greater freedom and flexibility in laying out designs and making structural fire protection decisions to ensure adequate fire safety.

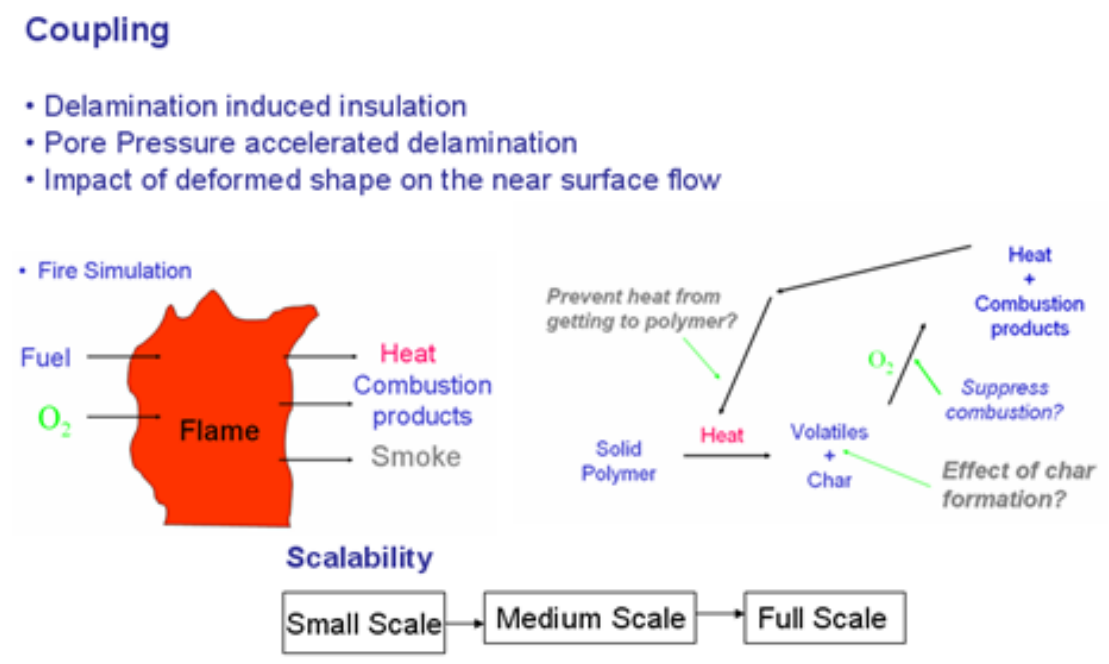

Fig. 1. Summary of problem challenges and the associated multi-physical processes.

Accurate modeling of thermal and mass diffusion through a 3-D structure is the critical first step in fire simulation and structural response prediction. To expedite the solution process for a large-scale structure with a fire structure coupling, an efficient fire dynamics simulator (FDS) developed by NIST [5-7] can be used to perform the fire simulation. FDS has been optimized by NIST for characterization of a low speed and thermally-driven flow, such as a fire. On the other hand, the Abaqus' FEM solver provides its great efficiency and versatility in solving a large-scale structure with both material and geometric nonlinearity [8]. The integration of these two commercial codes with customization can provide an ideal computational platform for simulation of the 3-D fire response and failure prediction of a large-scale structure component.

The fire response prediction of a loaded structural component requires fire and structure coupling at the interface between the CFD and structure domain. Several assumptions have been made in current fire simulation tools to predict the thermal response of a structure subjected to a fire exposure. For example, they often decouple the reacting flow processes from the response of structures which result in unreliable predictions of flame spread rate and overall thermal response of the structure. For a high fidelity fire response prediction, the interface coupling has to be established to exchange the information such as temperature, heat flux, and mass flux while satisfying the principles of mass and energy conservation [9]. While a two-way coupling between the fire and structure has been implemented in the FDS tool, the solid (thermal only) solver in FDS is not accurate due to the use of its 1-D heat transfer assumption. A solid object in FDS has been characterized as a group of 1-D column with their heat transfer along the column length direction. In general, it is difficult to determine a priori if the lateral heat exchange is important or not. Despite the limitation of the FDS tool in comparison with a fully capable CFD solver, it is still widely used to simulate the fire response of a large-scale structure because of its high computational efficiency with an acceptable level of accuracy.

To remove the limitation associated with the 1-D heat transfer modeling in the current FDS solver and characterize a general 3-D thermal and mass diffusion within a commercial FEM framework, an Abaqus 
Fire Interface Simulator Toolkit (AFIST) has been developed by packaging and integrating the solution modules in fire simulation, thermal decomposition, non-linear viscoelastic damage state evolution, thermalmechanical response prediction, and hybrid damage and failure prediction [10,11]. An overview of AFIST is shown in Fig. 2. The key GUI components in AFIST include: 1) generation of FDS and Abaqus model files based on users' input; 2) definition of model parameters for FDS; 3) definition of structural model parameters for Abaqus; 4) insertion of a coupling interface for co-simulation; and 5) display of cosimulation and fluid structural interaction results. Most recently, a unified coupling strategy for both thermal and mass coupling has been implemented in AFIST via Abaqus user defined subroutines. In addition, multiple coupling interfaces can be defined in AFIST for the structural response prediction subjected to fire.

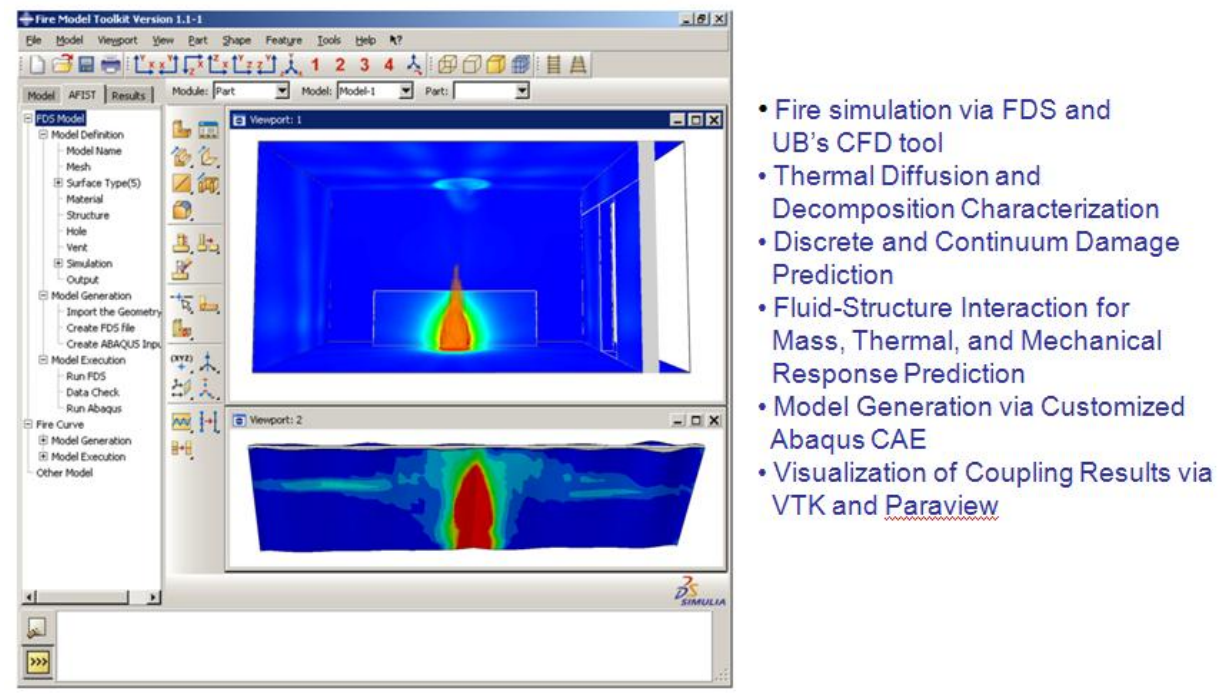

Fig. 2. Capability overview of AFIST.

\section{SUMMARY OF KEY SOLUTION MODULES IN AFIST'S THERMAL MASS COUPLING}

\section{A Customized FDS Solution Module}

Fire Dynamics Simulator (FDS) is a computational fluid dynamics model for simulating the fire-driven fluid flow [5]. It solves numerically a simplified form of the Navier-Stokes equations appropriate for lowspeed, thermally-driven flow, with an emphasis on smoke and heat transport from a fire. During our toolkit (AFIST) development, FDS has to be coupled with Abaqus to perform the fire simulation and to predict the resulting structure damage and final rupture. A comparison of the solution process between the customized FDS and its stand-alone version is shown in Fig. 3. In summary, the limitations associated with the standalone FDS are

- Unable to change the thermal boundary conditions during the solution progression;

- Ignorance of lateral heat transfer based on the 1-D thermal model for solids;

- Unable to apply a user-defined decomposition model; and

- No mechanical response analysis module. 


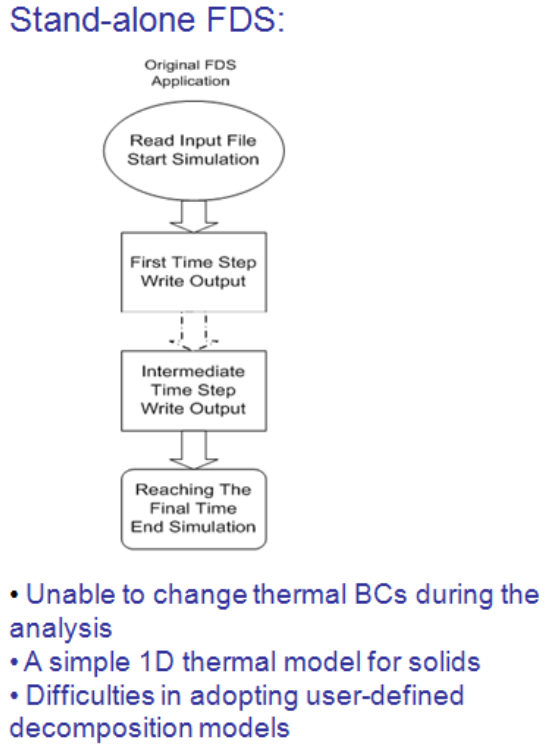

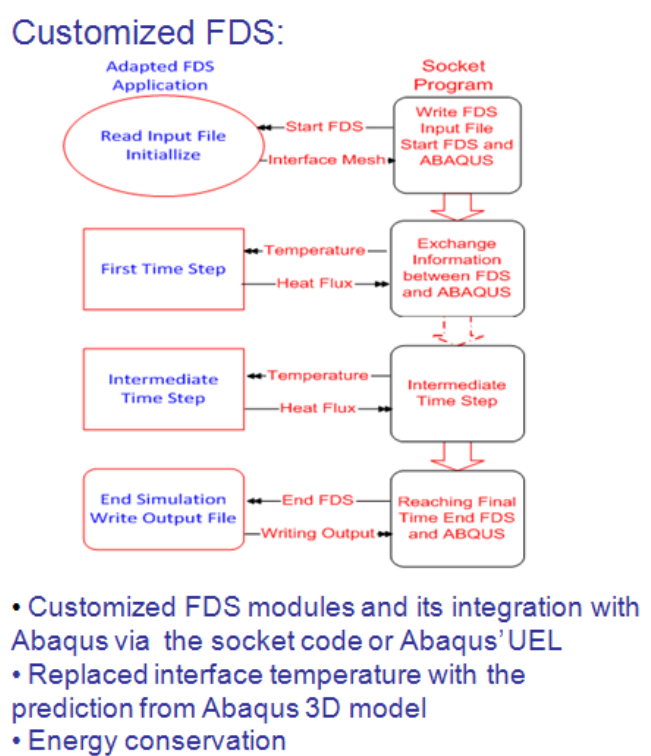

Fig. 3. Comparison of a stand-alone FDS and a customized FDS used in AFIST for its coupling with Abaqus.

These limitations are conquered in AFIST by customizing FDS and coupling it with the thermalmechanical damage model implemented in Abaqus. As shown in Fig. 3, the customized FDS allows the ability to define arbitrary thermal and mass boundary conditions at a coupling interface when an information exchange between FDS and Abaqus is invoked.

\section{Two-Way Coupling Between FDS and Abaqus for Mass and Thermal Transfer}

Under like the one-way coupling where a fire-induced thermal and mass diffusion problem is fully solved prior to an FEM-based structural analysis, a two-way coupling is implemented in AFIST where a 3-D thermal/mass diffusion problem is solved for the solid domain at a pre-defined coupling time using the boundary conditions given at the coupling interface. While the similar two-way coupling has been employed in the stand alone FDS, both the thermal and mass diffusion equations are solved by simplifying the solid domain as a 1-D structure. By replacing the 1-D thermal and mass diffusion solver in FDS with its corresponding 3-D solution modules in Abaqus, it can not only capture the lateral hear and mass transfer but also define a new thermal decomposition and mass diffusion model through the user-defined defined solution models in Abaqus. A summary of two-way coupling implemented in AFIST for both the mass and heat transfer is illustrated in Fig. 4.

As shown in Fig. 4, the mass flux ( $\left.\dot{m}^{\prime \prime}\right)$ of decomposed gas computed by Abaqus at the interface is used as the boundary condition for solving the fluid equations of the FDS domain. The pressure $(P)$, in return, is used subsequently as the boundary condition for the Abaqus domain solving the internal decomposed gas pressure. The same solution strategy is applied for the heat transfer where the information exchange for the mass flux and pressure used in the mass transfer is replaced by the temperature and heat flux. To demonstrate the validity of the implemented two-way coupling, we applied the AFIST toolkit for a 1-D mass and heat transfer problem where the predictions can be directly compared with the corresponding FDS prediction for the verification study. Linear interpolation is applied to map the temperature from Abaqus to FDS with different mesh densities. As can be seen from Fig. 4, an excellent agreement has been achieved in prediction of the time history of both the mass flux and temperature. 
Mass Transfer Coupling

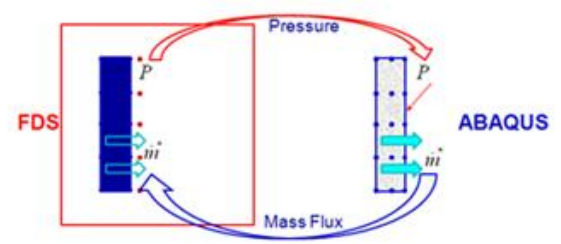

Mass Transfer Equation in FDS, where $Y_{i}$ is defined as mass fraction of gas specie $i$

$\rho \frac{D Y_{i}}{D t}=\nabla \cdot\left(\rho D \nabla Y_{i}\right)$

At the interface, the mass flux is specified by the values passed in from ABAQUS.

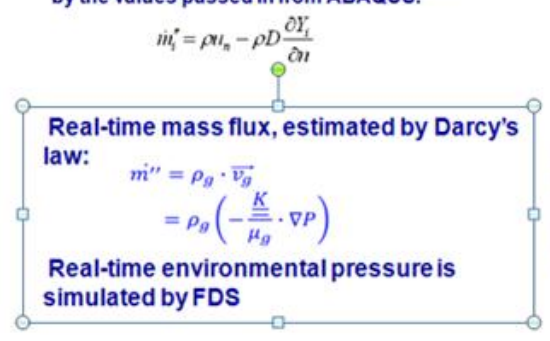

Heat Transfer Coupling

FDS

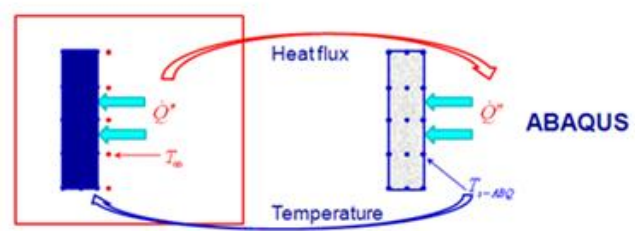

- Real-time heat flux:

$$
\dot{Q}^{\prime \prime}=\varepsilon \sigma\left(T_{\infty}^{4}-T_{s-A B Q}^{4}\right)+h_{c o n v}\left(T_{\infty}-T_{s-A B Q}\right)
$$

- Real-time surface T:

Precisely simulated $T_{s-A B Q}$ using thermal decomposition model

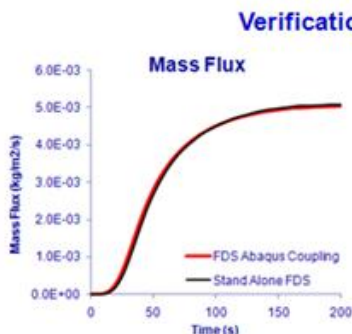

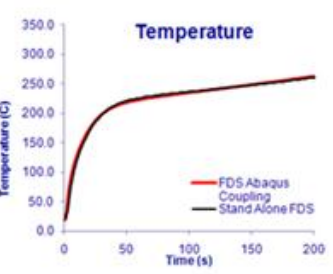

Fig. 4. Illustration of two-way coupling for mass and heat transfer.

\section{Unified Implementation of Two-Way Coupling in AFIST}

A direct coupling between a user-defined CFD solver and Abaqus' structural solver can be achieved via Abaqus' inherent co-simulation framework. Five key solution steps during the coupling analysis are: 1) generation of structural grids for the fluid domain within Abaqus; 2) transformation of the temperature on the solid boundary from Abaqus to the CFD solver; 3) transformation of the heat flux from FDS to Abaqus at the structural boundary; and 4) performance of the sub-cycling between the FDS and Abaqus solver. The use of the existing co-simulation framework provided by Abaqus is not applicable for the mass transfer through the two-way coupling. This is mainly due to that current Abaqus co-simulation interface does not support transformation of physical variables such as pressure and mass fractions between the Abaqus and CFD solver. To circumvent this difficulty, a user defined element approach is used for the two-way coupling of the mass transfer. As shown in Fig. 5, a layer of user-defined 2-D membrane elements are attached at the coupling interface and they share the same nodes of the underlying 3-D solid elements. The Dirichlet pressure boundary conditions (obtained from the FDS domain) are enforced at the interface via the penalty method. 
Previous coupling strategy via co-simulation (heat transfer coupling only):

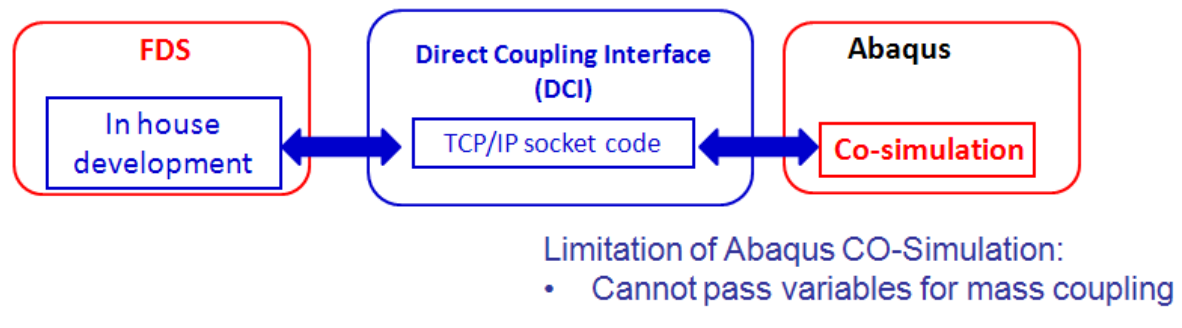

Current coupling strategy via user element (both mass and heat transfer coupling):

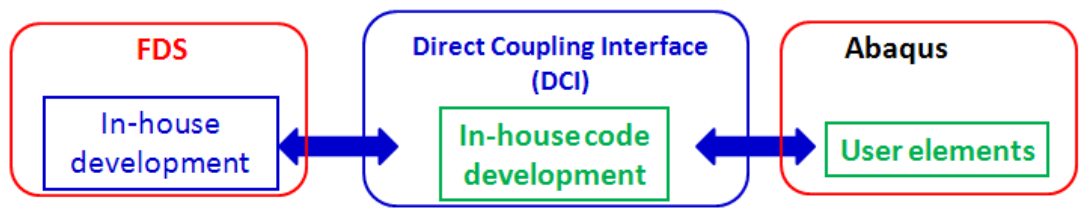

Fig. 5. Illustration of new coupling strategy used in AFIST for mass and heat transfer through user defined elements.

Because of the use of a distinct mesh in the fluid and solid domain, a conservation of mass and energy has to be forced over the spatial and temporal domain. Since local conservation being enforced on each element is computationally expensive, we have implemented a global approach to apply the conservation principle for the coupling surface. First, the variable (mass flux or heat flux) over a local domain is determined from the numerical interpolation. Next, a scaled factor is applied on its total quantity to ensure that the total mass and energy have been conserved.

\section{Implementation of Multiple Coupling Interfaces in AFIST}

Both the mass and thermal coupling described before may have to be enforced on a system with multiple coupling interfaces, such as in a room corner fire or a 3-D object embedded within a fuel source. A multiinterface coupling scheme has been implemented in AFIST. This scheme takes the advantage of Fortran 90's new feature, namely, the derived data types. Derived data types in Fortran 90 are similar to the concept of class in $\mathrm{C}++$, through which different data belonging to the same object can be encapsulated into one group. In our multi-interface coupling, the data belonging to one interface is grouped into one object and the interface mapping and data exchange are processed for each interface. A flow chart for the implementation of the multiple coupling interfaces is shown in Fig. 6. 


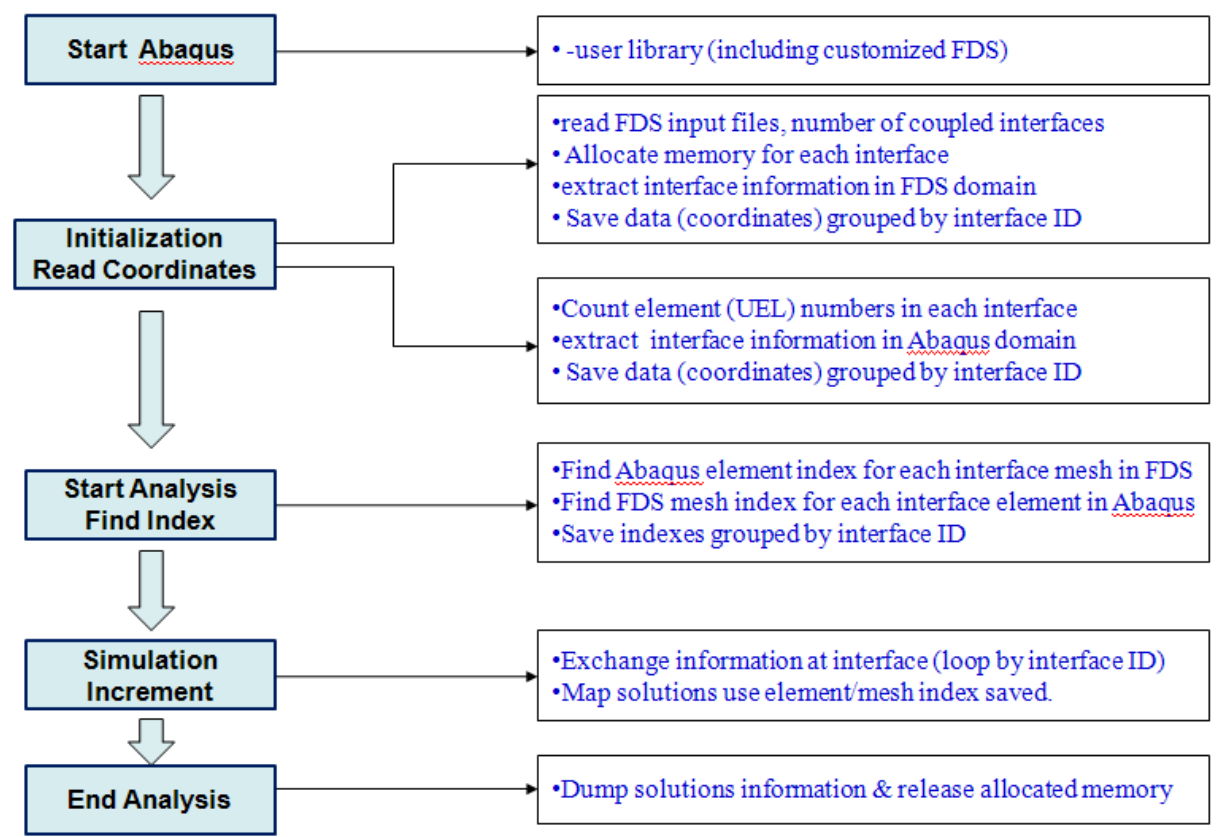

Fig. 6. Summary of solution procedures used in the multi-interface coupling.

\section{EXAMPLE APPLICATIONS AND VALIDATIONS}

A suite of demonstration and verification examples are used below to demonstrate the applicability and validity of AFIST for fire simulation and thermal response prediction of a structural component.

\section{Verification of Mass and Thermal Coupling in AFIST Using FDS for 1-D Simulation}

Four verification tests have been performed for the mass transfer coupling using the stand alone FDS and our AFIST toolkit with the verification strategy shown in Fig. 7. They are:

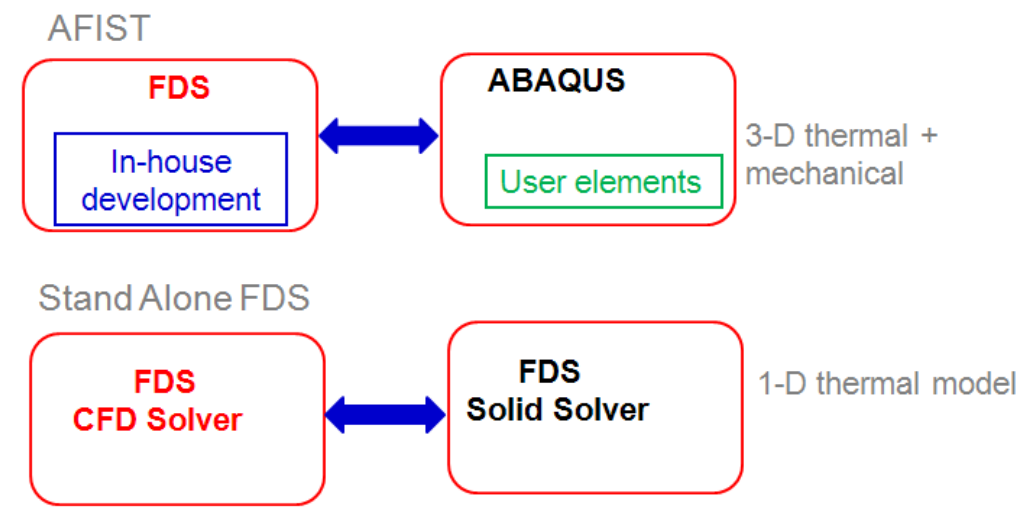

Simplifying Abaqus thermal decomposition model to match FDS internal solid solver.

Fig. 7. Illustration of verification strategy using the stand-alone FDS solver.

- Test 1 - fire simulation with $100 \%$ non-combustible decomposed gas using AFIST's single component and the stand alone FDS tool;

- Test 2 - fire simulation with $100 \%$ combustible decomposed gas using AFIST's single component and the multi-component mass coupling solution module;

- Test 3 - fire simulation with a mixture of $90 \%$ combustible and $10 \%$ non-combustible decomposed gas using both AFIST and FDS tool; and 
- Test 4 - fire simulation with a mixture of $60 \%$ combustible and $40 \%$ non-combustible decomposed gas using both AFIST and FDS tool.

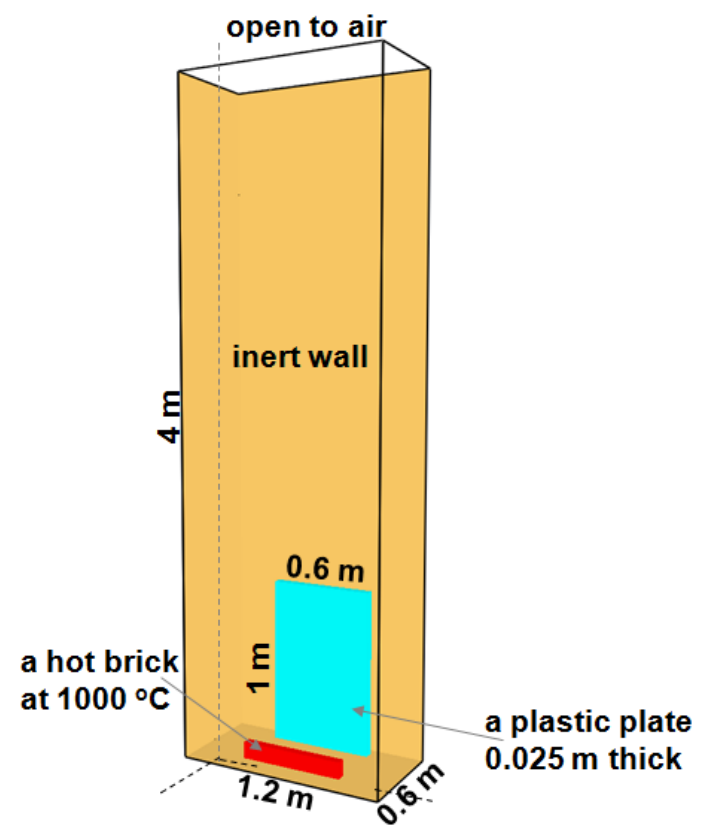

Fig. 8. Problem statement for verification cases.

Table 1. Thermal decomposition properties of the plastic plate.

\begin{tabular}{|l|r|}
\hline \multicolumn{1}{|c|}{ Properties } & \multicolumn{1}{c|}{ Value } \\
\hline$A_{s g}\left(\mathrm{~s}^{-1}\right)$ & 3015730.0 \\
\hline$E_{a}(\mathrm{~J} / \mathrm{kg} \cdot \mathrm{mol})$ & 23900.0 \\
\hline$n(-)$ & 1.0 \\
\hline$h(\mathrm{~J} / \mathrm{kg})$ & $2.0 \times 10^{13}$ \\
\hline$\rho_{i}\left(\mathrm{~kg} / \mathrm{m}^{3}\right)$ & 1180.0 \\
\hline$\rho_{f}\left(\mathrm{~kg} / \mathrm{m}^{3}\right)$ & 118.0 \\
\hline$c_{p}(\mathrm{~J} / \mathrm{kg} \cdot \mathrm{K})$ & 1900.0 \\
\hline$k(\mathrm{~W} / \mathrm{m} \cdot \mathrm{K})$ & 0.17 \\
\hline
\end{tabular}

The problem statement associated with these four test cases is defined in Fig. 8. A plastic plate is placed in a room with its front and top faces open to the air and with inert walls on all the other sides. The room is $1.2 \mathrm{~m}$ long, $0.6 \mathrm{~m}$ wide and $4.0 \mathrm{~m}$ tall. The plate is $1.0 \mathrm{~m}$ high, $0.6 \mathrm{~m}$ wide and $0.025 \mathrm{~m}$ thick. A hot brick of $1000{ }^{\circ} \mathrm{C}$ placed in front of the plate is used as a heat source. The plate absorbs the radiation energy from the hot brick and undergoes thermal decomposition. The decomposition gas is released and dispersed in the room. The reaction rates are functions of local mass concentration and temperature, and calculated as a combination of the Arrhenius and power function defined as,

$\frac{d \rho}{d t}=-\rho_{i}\left(\frac{\rho}{\rho_{i}}\right)^{n} A \exp \left(\frac{-E}{R T}\right)$

A comparison of the time history of temperature and mass flux predicted from Test 1 at a selected location is shown in Fig. 9. Identical results are obtained indicating that the multi-component mass coupling has been implemented correctly. The accuracy of prediction for Test Case 2, 3, and 4 is demonstrated in Figs. 10, 11, and 12, respectively. Both the temperature and mass flux are in an excellent agreement with the prediction from using the stand alone FDS solver for both fully combustible and mixed combustible and non-combustible gas. 


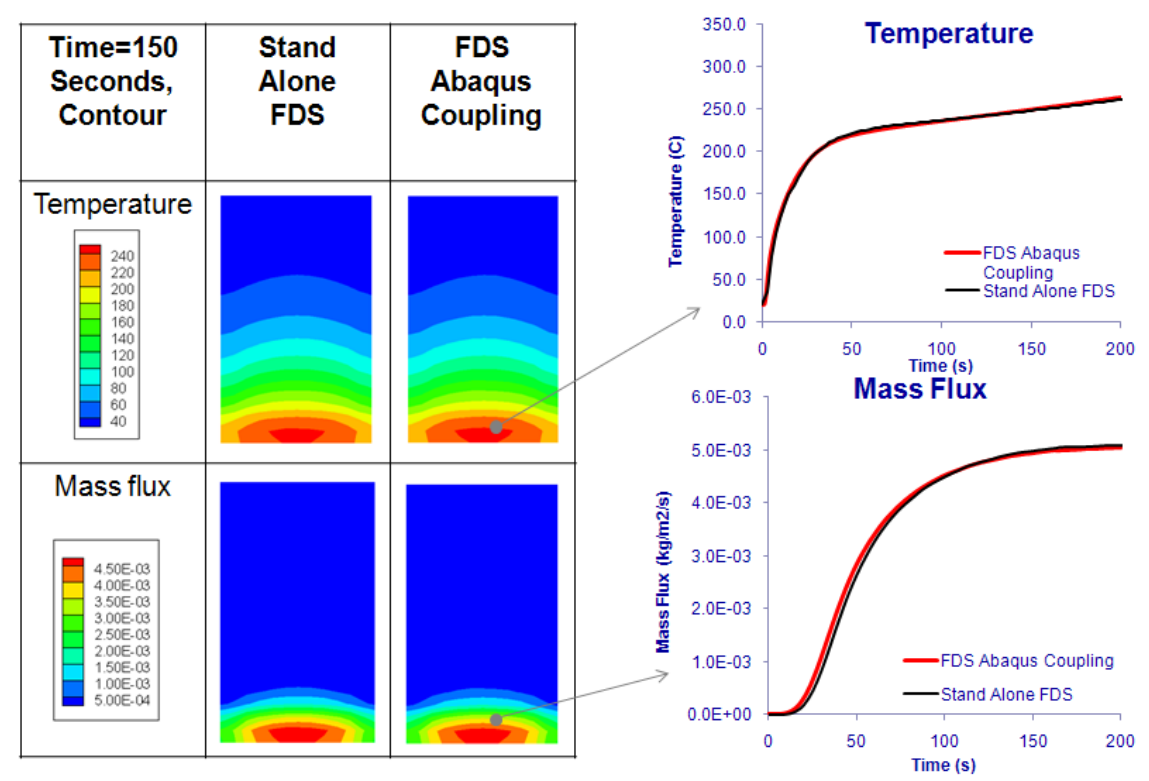

Fig. 9. Verification results for Test Case 1.

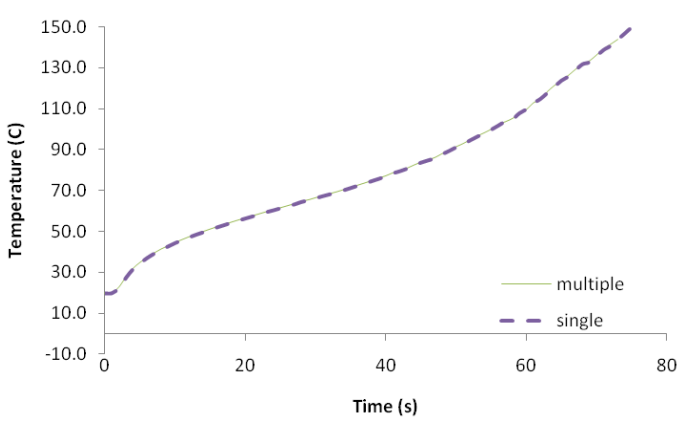

(a)

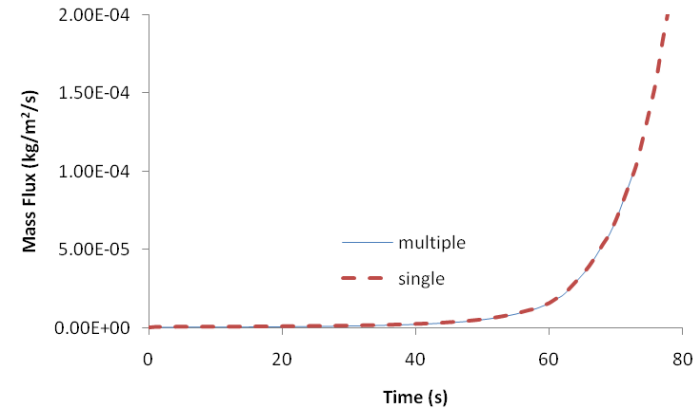

(b)

Fig. 10. Verification results for Test Case 2: (a) temperature; (b) mass flux.

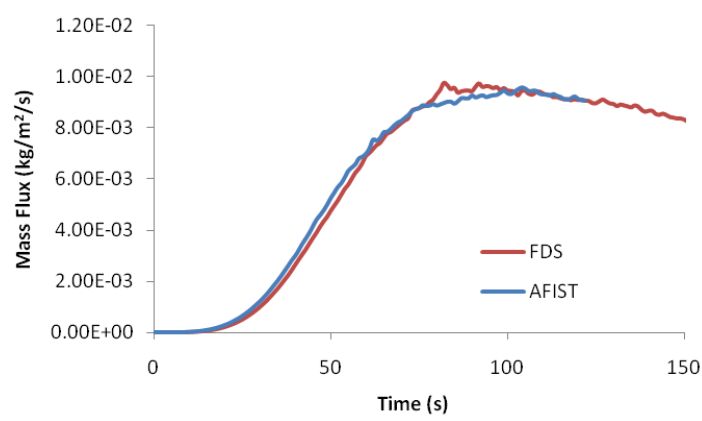

(a)

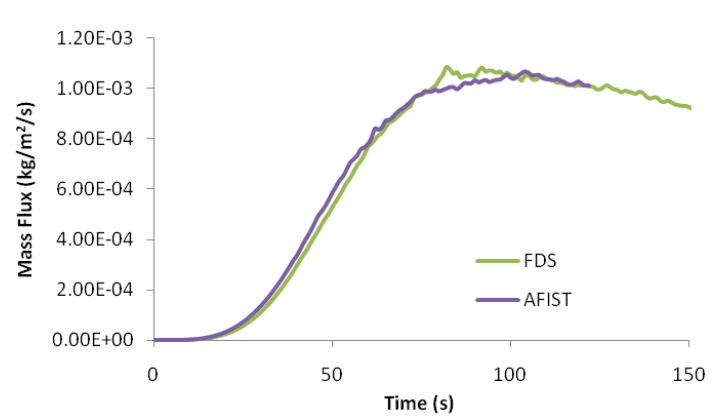

(b)

Fig. 11. Verification results for Test Case 3: (a) fuel mass flux; (b) non-combustible mass flux. 


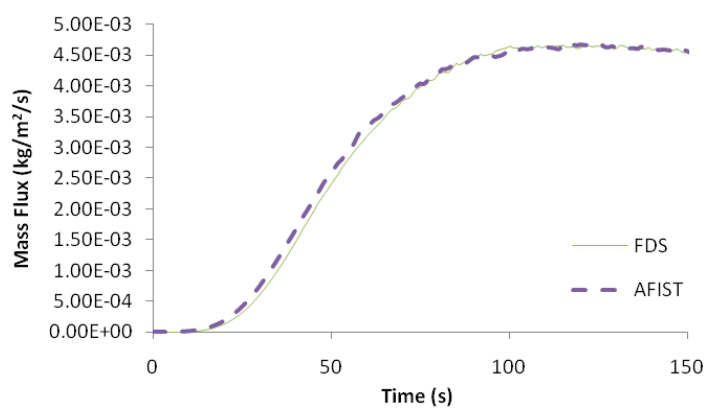

(a)

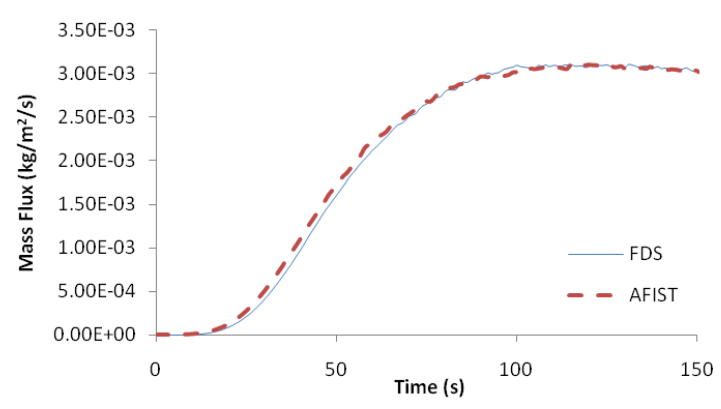

(b)

Fig. 12. Verification results for Test Case 4: (a) fuel mass flux; (b) non-combustible mass flux.

\section{Exploration of Flame Spread Using AFIST}

The flame spread phenomena has been simulated by AFIST as shown in Fig. 13. The problem set up is the same as depicted in Fig. 8, except that the plate is $3.0 \mathrm{~m}$ high and the decomposition gas is combustible. The decomposition gas is $\mathrm{C}_{5} \mathrm{H}_{8} \mathrm{O}_{2}$ with heat of combustion of $23900.0 \mathrm{~kJ} / \mathrm{kg}$, as shown in Table 2. The distribution of the plate temperature at three different times is shown in Fig. 14. The burning rate, heat release rate, and smoke dispersion at these three different times are given in Fig. 15. Clearly we can see that the flame has propagated upward indicating the flame spread phenomena. Initially, only the bottom of the plate is subjected to the decomposition due to the radiation heat from the hot brick. Then the decomposed gas is released from the bottom and contributed to the fire in the CFD domain. The burned gas which has a higher temperature and lower density flows upward due to buoyancy forces. The material is further decomposed under the additional heating. The distribution of the burning rate is shown in Fig. 15 indicating the occurrence of the flame spread event

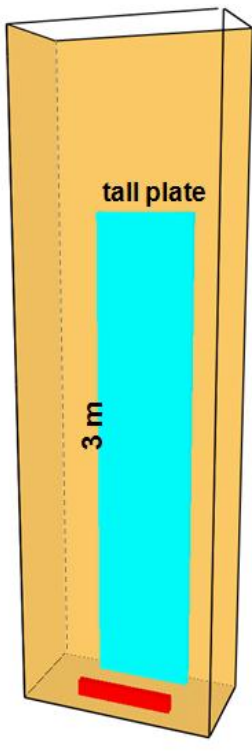

Fig. 13. Problem statement for the flame spread.

Table 2. Combustion properties of the decomposed gas.

\begin{tabular}{|l|r|}
\hline \multicolumn{1}{|c|}{ Properties } & \multicolumn{1}{c|}{ Value } \\
\hline Chemical formula & $\mathrm{C}_{5} \mathrm{H}_{8} \mathrm{O}_{2}$ \\
\hline Heat of combustion $(\mathrm{kJ} / \mathrm{kg})$ & 23900.0 \\
\hline Soot yield (-) & 0.022 \\
\hline
\end{tabular}



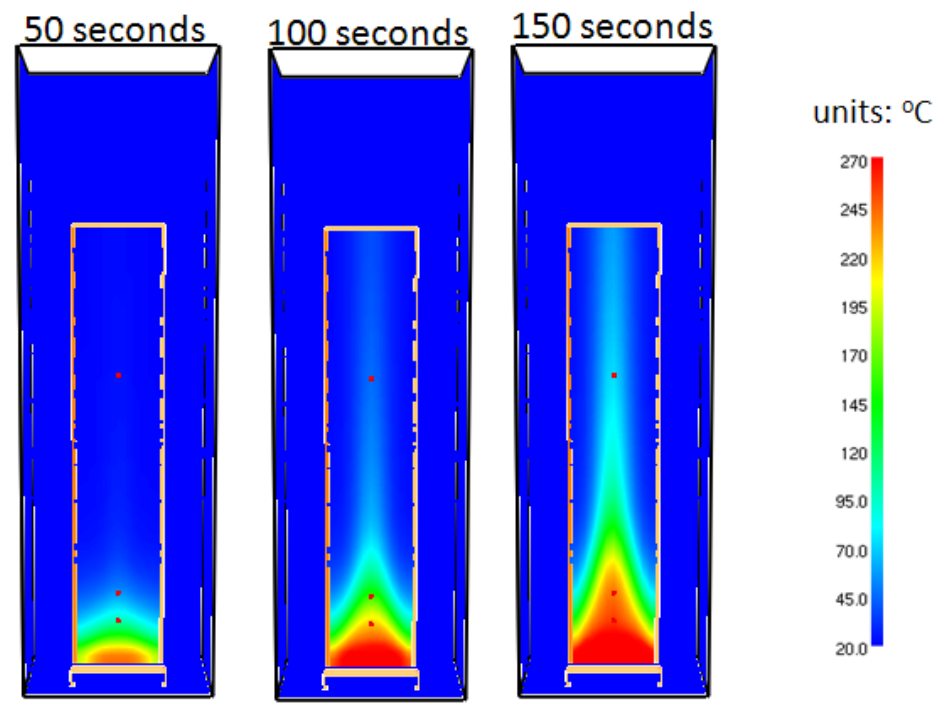

Fig. 14. Flame spread phenomena, temperature plot.
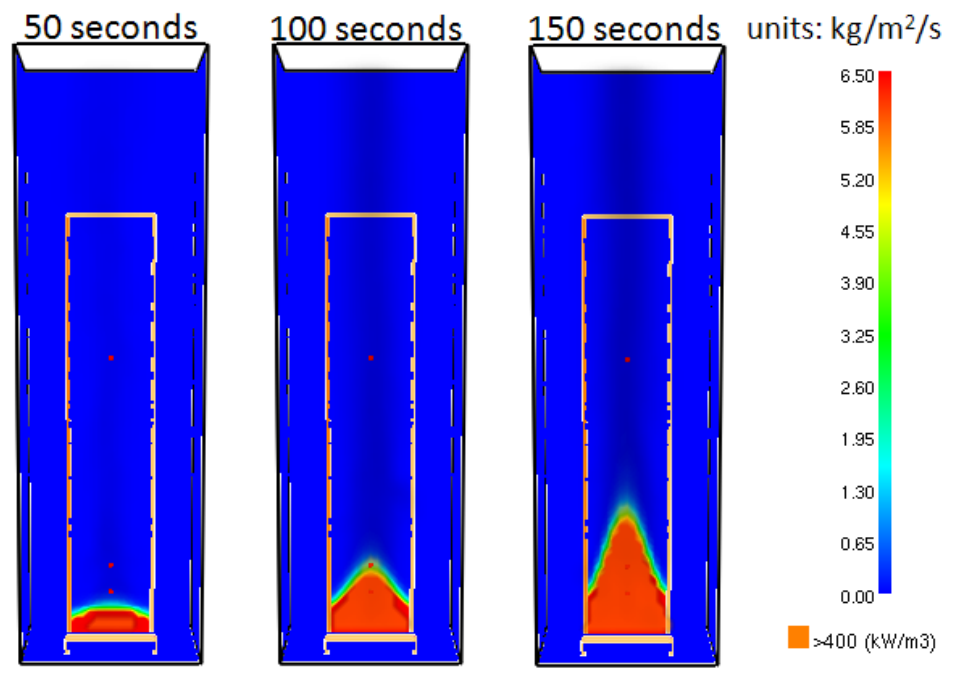

Fig. 15. Flame spread phenomena, burning rate plot.

\section{AFIST Validation Using a Steel Tube Subjected to a Room Corner Heating}

This example has been used in the FDS validation manual given by Refs. [6,12]. As shown in Fig. 16, a steel tube filled with an insulation material is hung on the ceiling of a room. A burner is placed in the room corner with its heating rate of $5000 \mathrm{~kW} / \mathrm{m}^{2}$. Using the AFIST coupling solver, the gas temperature, room temperature, and the temperature of the steel tube are predicted and compared with the experimental measurement. A comparison of the time history of the temperature at three pre-selected locations of the steel tube is presented in Fig. 17. Good agreement between the AFIST prediction and test data has been achieved indicating the validity of the FDS and Abaqus coupling. 


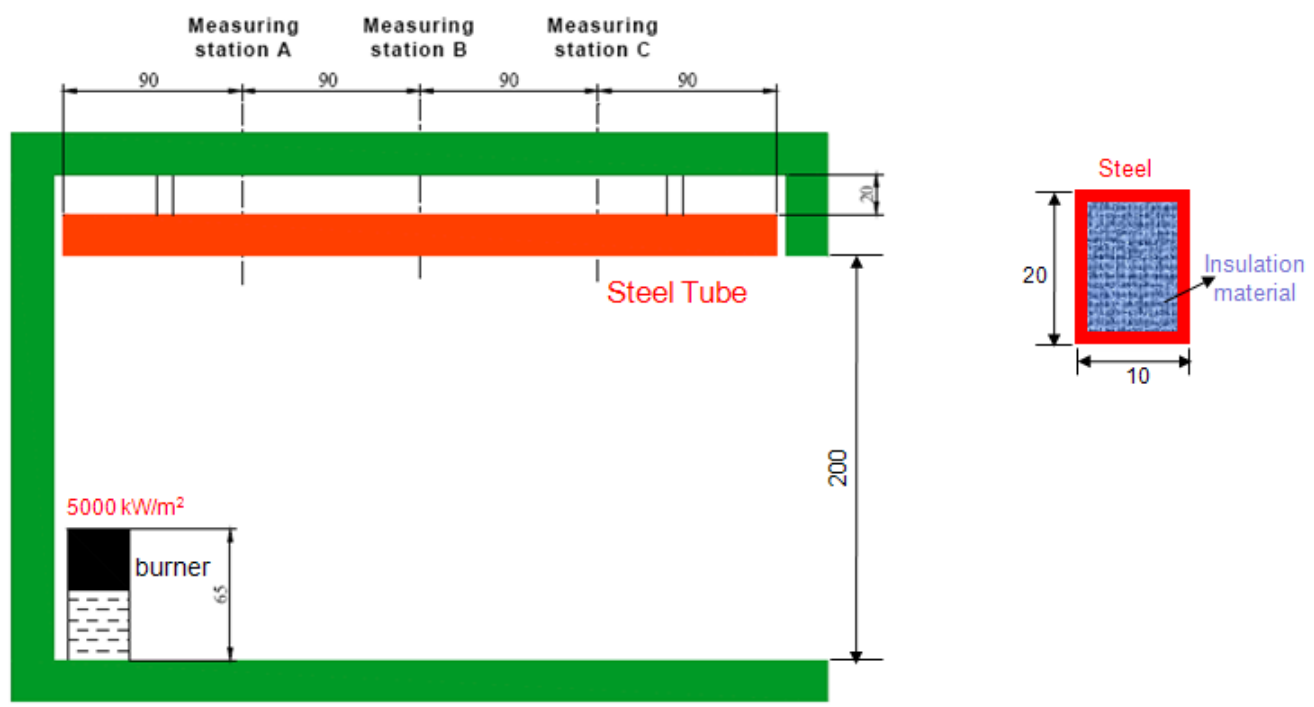

The width is the same as the height $(240 \mathrm{~cm})$

Fig. 16. A steel tube subjected to a room corner heating [12].
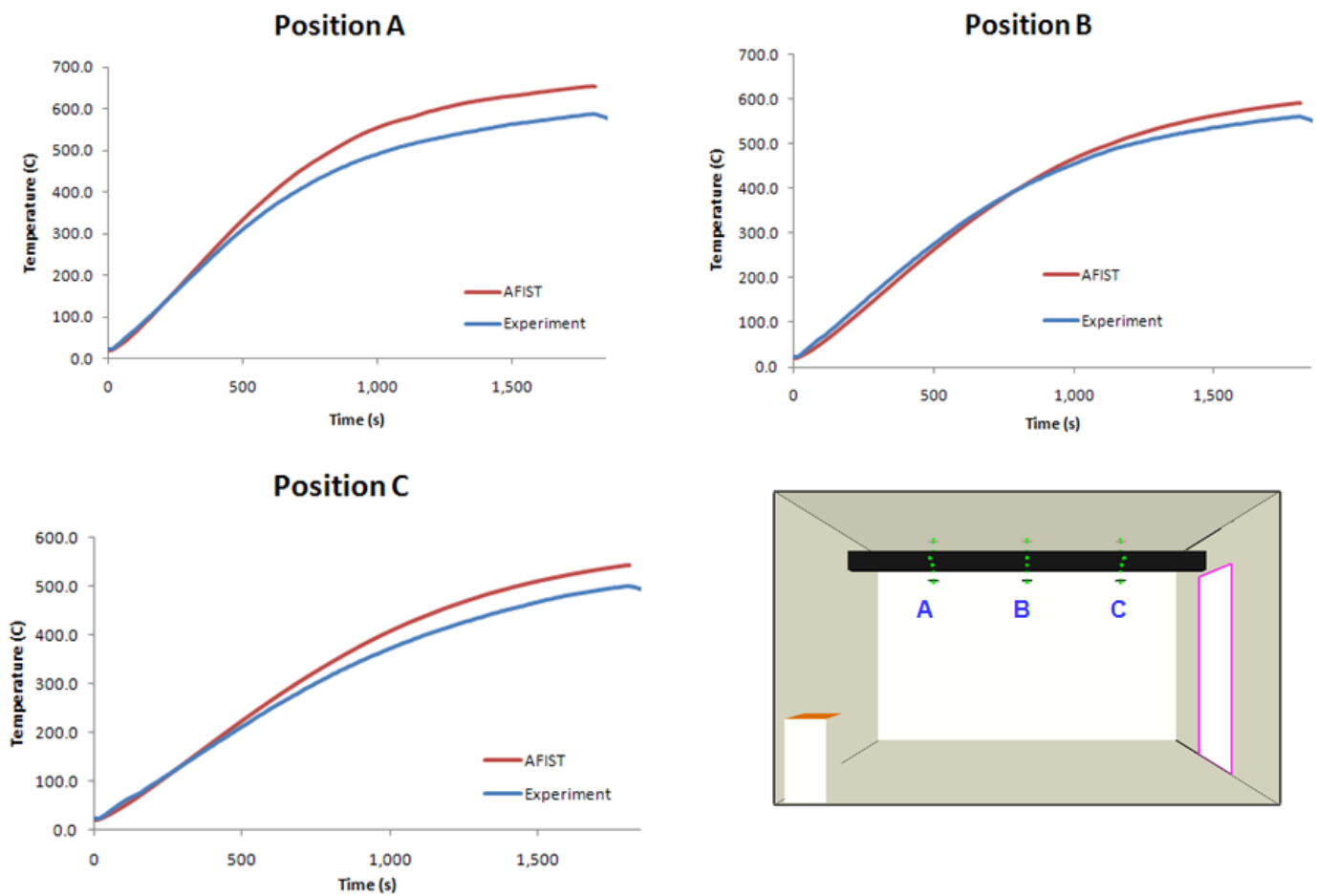

Fig. 17. Comparison of time history of temperature at three locations $(A, B, C)$.

\section{SUMMARY AND CONCLUSIONS}

Given the limitation from the sequential (or one-way) coupling between a fire simulator and a structural analyzer, a two-way coupling has been developed and implemented in AFIST via its co-simulation framework. Validation and example application of the two-way coupling have been performed to 1) explore the effect of the mixed gas on the fire response and temperature distribution; 2) explore the flame spread phenomena; 3) verify the implemented coupling strategy using the stand alone FDS solver; and 4) validate the accuracy at structural level by comparing the simulation results with the test data. 
In addition to the thermal coupling implemented in AFIST, the mass coupling has also been included in order to evaluate the amount of the decomposed gas, the resulting pore pressure, and the property of the decomposed gas such as combustibility and toxicity. Since the existing co-simulation framework in Abaqus is not applicable for transferring additional physical variables associated mass coupling, a user-defined interface element approach has been developed to form a unified approach for both the mass and thermal coupling.

\section{ACKNOWLEDGMENT}

This research has been supported by the Office of Naval Research (ONR) under grant N00014-08-C-0591 with Dr Luise Couchman as the program monitor.

\section{REFERENCES}

[1] Mouritz, A.P., and Mathys, Z., (1999) Post-fire mechanical properties of marine polymer composites, Composite Structures, 47: 643-653. http://dx.doi.org/10.1016/S0263-8223(00)00043$\underline{\mathrm{X}}$

[2] Mouritz, A.P., and Mathys, Z., (2001) Post-fire mechanical properties of glass-reinforced polyester composites, Composites Science and Technology, 61: 475-490. http://dx.doi.org/10.1016/S0266-3538(00)00204-9

[3] Mouritz, A.P., E. Geller, P.Burchill and Challis, K., (2001) Review of advanced composite structures for naval ships and submarines,' Composite Structures, 53: 21-41. http://dx.doi.org/10.1016/S0263-8223(00)00175-6

[4] Mouritz, A.P., Feih, S., Kandare, E., Mathys, Z., Gibson, A.G., DesJardin, P.E., Case, S.W., and Lattimer, B.Y., (2009) Review of fire structural modeling of polymer composites, Composites Part A, 40, (12): 1800-1814. http://dx.doi.org/10.1016/j.compositesa.2009.09.001

[5] McGrattan, K.B., Hostikka, S., Floyd, J.E., Baum, H.R., and Rehm, R.G. Fire Dynamics Simulator (Version 5), Technical Reference Guide, Volume 1: Mathematical Model. NIST Special Publication 1018.5, National Institute of Standards and Technology, Gaithersburg, Maryland, October 2007.

[6] McGrattan, K.B., Hostikka, S., Floyd, J.E., and Klein, B. Fire Dynamics Simulator (Version 5), Technical Reference Guide, Volume 3: Validation. NIST Special Publication 1018.5, National Institute of Standards and Technology, Gaithersburg, Maryland, October 2007.

[7] McGrattan, K.B., Klein, B.W., Hostikka, S., and Floyd, J.E. Fire Dynamics Simulator (Version 5), User's Guide. NIST Special Publication 1019.5, National Institute of Standards and Technology, Gaithersburg, Maryland, October 2007.

[8] SIMULIA/Abaqus, 'Abaqus user's manual, version 6.10 edition,' (2010).

[9] Luo. C., Xie, W., and Desjardin, P.E., (2010) Fluid-Structure Simulations of Composite Material Response for Fire Environments, Fire Technology (in press). http://dx.doi.org/10.1007/s10694$\underline{009-0126-4}$

[10] Luo, C., Chen, L., Lua, J., and Shi, J., "A 3D ABAQUS toolkit for thermal-mechanical damage prediction of composite sandwich structures subjected to fire," $50^{\text {th }}$ AIAA/ASME/ ASCE/AHS/ASC Structures, Structural Dynamics, and Materials Conference 2009, Palm Springs, CA, May 04-07 (2009).

[11] Luo, C., Chen, L., Lua, J., and Liu, P., "Abaqus fire interface simulator toolkit (AFIST) for coupled fire and structural response prediction," $51^{\text {st }}$ AIAA/ASME/ASCE/AHS/ASC Structures, Structural Dynamics, and Materials Conference 2010, Orlando, FL, April 12-15 (2010).

[12] Wickström, U., Jansson, R., and Tuovinen, H., "Validation fire tests on using the adiabatic surface temperature for predicting heat transfer," Fire Technology, SP Report 2009, 19, ISBN 978-9186319-03-8. 